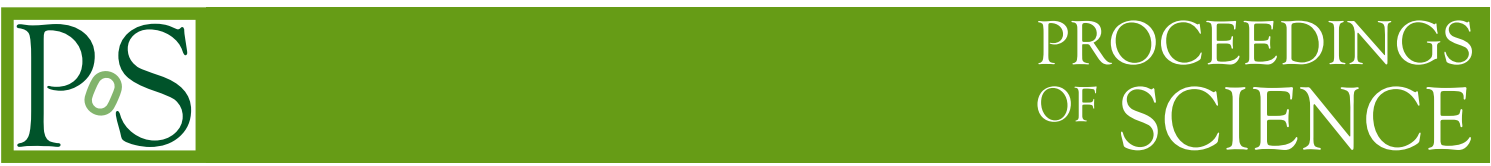

\title{
Aspects of QCD Vacuum Structure
}

\author{
Peter J. Moran and Derek B. Leinweber* \\ Special Research Centre for the Subatomic Structure of Matter and Department of Physics, \\ University of Adelaide, SA 5005, Australia \\ E-mail: peter.moran@adelaide.edu.au, \\ dleinweb@physics.adelaide.edu.au
}

\begin{abstract}
The impact of dynamical fermions on the vacuum structure of QCD is explored. Of particular interest is the topological charge correlator, $\langle q(x) q(0)\rangle$, where negative values at small $x$ reveal a sign-alternating layered structure to the topological-charge density of the QCD vacuum. We consider large $28^{3} \times 96$ lattices from the MILC collaboration, and develop a new gluonic definition of the topological charge density, founded on a new over-improved stout-link smearing algorithm. The algorithm reproduces established results from the overlap formalism and is designed to preserve instantons. We examine the extent to which instanton-like objects are found on the lattice. Finally, we investigate the effects of dynamical sea-quark degrees of freedom on topology and find that the magnitudes of the negative dip in the $\langle q(x) q(0)\rangle$ correlator and the positive $\left\langle q(0)^{2}\right\rangle$ contact term are both increased with the introduction of dynamical fermion degrees of freedom. This is in accord with expectations based on charge renormalization and the vanishing of the topological susceptibility in the chiral limit.
\end{abstract}

The XXV International Symposium on Lattice Field Theory

July 30-4 August 2007

Regensburg, Germany

${ }^{*}$ We thank the Australian Partnership for Advanced Computing (APAC) and the South Australian Partnership for Advanced Computing (SAPAC) for generous grants of supercomputer time which have enabled this project. This work is supported by the Australian Research Council. 


\section{Introduction}

Understanding the topological structure of the QCD vacuum remains a central focus of modern Lattice QCD studies. For computational reasons, most previous studies have focused on gauge fi elds generated using the quenched approximation. In the following proceedings, we present a quantitative comparison of vacuum structure for quenched and dynamical-fermion gauge fi elds.

The introduction of fermion loops into the QCD action renormalizes the coupling and demands smaller values for $\beta$ in obtaining the same lattice spacing, $a$. Smaller $\beta$ values will admit rougher gauge fi elds such that we expect to see a higher density of non-trivial topological excitations, particularly for lighter sea-quark masses. For example, we anticipate larger values for the meansquare topological charge density $\left\langle q^{2}(x)\right\rangle_{x}$. This combined with the vanishing of the topological susceptibility in the chiral limit leads to our prediction that the negative dip in the topological charge density correlator, $\langle q(x) q(0)\rangle$, will be enhanced in full QCD with light dynamical-fermions. To the best of our knowledge, this is the fi rst study of the $\langle q(x) q(0)\rangle$ correlator in full QCD.

In order to study these differences in vacuum structure on the very large MILC lattices, we commence with the development of a new gluonic defi nition of the topological charge density, founded on a new form of over-improved [1] stout-link smearing [2] algorithm, designed to stabilize instantons. We then examine the extent to which instanton-like objects are found on the lattice. Finally, we investigate the effects of dynamical sea-quark degrees of freedom on topology and fi nd that the magnitudes of the negative dip in the $\langle q(x) q(0)\rangle$ correlator and the positive $\left\langle q(0)^{2}\right\rangle$ contact term are both increased with the introduction of dynamical fermion degrees of freedom. The effect is signifi cant and is easily observed in the visualizations of the topological charge density provided at the close of these proceedings.

\section{Over-Improved Stout-Link Smearing}

The removal of short-distance UV fluctuations is an important aspect of defi ning the topological charge of a rough gauge-fi eld confi guration. For gluonic topological charge operators, one often applies iterative smoothing algorithms which hold the risk of destroying the very structures one hopes to reveal. The corrosion of topological excitations in the QCD vacuum under smoothing is due to the presence of discretization errors in the approximation of the action. In the past there have been attempts to remove these errors via the combination of different sized Wilson loops in the calculation of the local action. When combining these loops, one must carefully choose the coeffi cients of the different shapes in order to cancel the leading order error terms, thereby resulting in an improved action [3, 4].

Despite the improvements, improved actions can still spoil instantons [5]. Consider, for example the Symanzik $O\left(a^{2}\right)$ improved action, composed of the plaquette $\left(P_{\mu \nu}\right)$ and rectangular $\left(R_{\mu \nu}, R_{\nu \mu}\right)$ Wilson loops.

$$
S_{S}=\beta \sum_{x} \sum_{\mu<v}\left[\frac{5}{3}\left(1-P_{\mu v}(x)\right)-\frac{1}{12}\left(\left(1-R_{\mu v}(x)\right)+\left(1-R_{v \mu}(x)\right)\right)\right]
$$


We can Taylor expand the Symanzik action in orders of $a$ and following Perez, et al. [1] substitute the classical instanton solution [6]

$$
A_{\mu}(x)=\frac{x^{2}}{x^{2}+\rho^{2}}\left(\frac{i}{g}\right) \partial_{\mu}(S) S^{-1}, \quad S \equiv \frac{x_{4} \pm i \vec{x} \cdot \vec{\sigma}}{\sqrt{x^{2}}},
$$

into the expanded action to fi nd

$$
S_{S}^{i n s t}=\frac{8 \pi^{2}}{g^{2}}\left[1-\frac{17}{210}\left(\frac{a}{\rho}\right)^{4}\right] .
$$

The negativity of the $O\left(a^{4}\right)$ error means that this action will destabilize instantons when used in an iterative scheme. This occurs because the smoothing algorithms are designed to remove action and will do so by effectively reducing $\rho$ to obtain a lower action. Eventually the instantons become suffi ciently small that discretization errors allow them to be removed from the lattice.

Perez, et al. [1] proposed that instead of combining different loop combinations in order to suppress the discretization errors, they could instead tune their coeffi cients such that the errors became positive for a classical instanton. By doing this, instantons should be stable under cooling. We extend their work, using a plaquette plus rectangle action, in the interests of locality, and modern stout-link smearing [2].

Taking the Symanzik action (2.1) and introducing a new parameter $\varepsilon$, such that $\varepsilon=1$ provides the Wilson action and $\varepsilon=0$ provides the Symanzik-improved action, implies the following form for the over-improved action

$$
S(\varepsilon)=\beta \sum_{x} \sum_{\mu<v}\left[\frac{5-2 \varepsilon}{3}\left(1-P_{\mu v}(x)\right)-\frac{1-\varepsilon}{12}\left(\left(1-R_{\mu v}(x)\right)+\left(1-R_{v \mu}(x)\right)\right)\right] .
$$

Taylor expanding this action for the classical instanton solution, one fi nds that for $\varepsilon<0$ the leading order $a^{2}$ errors are positive

$$
S^{i n s t}(\varepsilon)=\frac{8 \pi^{2}}{g^{2}}\left[1-\frac{\varepsilon}{5}\left(\frac{a}{\rho}\right)^{2}+\frac{14 \varepsilon-17}{210}\left(\frac{a}{\rho}\right)^{4}\right] .
$$

The question is now: How negative should $\varepsilon$ be? To answer this, we propose the following method.

Given $S(\varepsilon)$, select some value of $\varepsilon$ and plot $S(\varepsilon) / S_{0}$ as a function of $\rho$. Ideally this will result in a straight line at $S(\varepsilon) / S_{0}=1$. What we actually observe is illustrated in Fig. 1. Note that it is the value of the slope of the curve that is important when deciding how an instanton will change under a given smoothing algorithm. Varying $\varepsilon$ results in curves of varying slope. We settled on a value of $\varepsilon=-0.25$ as providing a nice result.

\section{Vacuum Structure}

With the over-improved stout-link smearing procedure completely defi ned we now proceed to perform a study of topological excitations in the QCD vacuum. We also provide a few results obtained using a 3-loop improved cooling algorithm [4]. We use one set of quenched gauge fi elds and two sets of dynamical gauge fi elds in order to investigate the effect of dynamical sea quarks and varying quark mass. The gauge fi elds were generated by the MILC collaboration [7, 8], and their details are summarized in Table 1. 


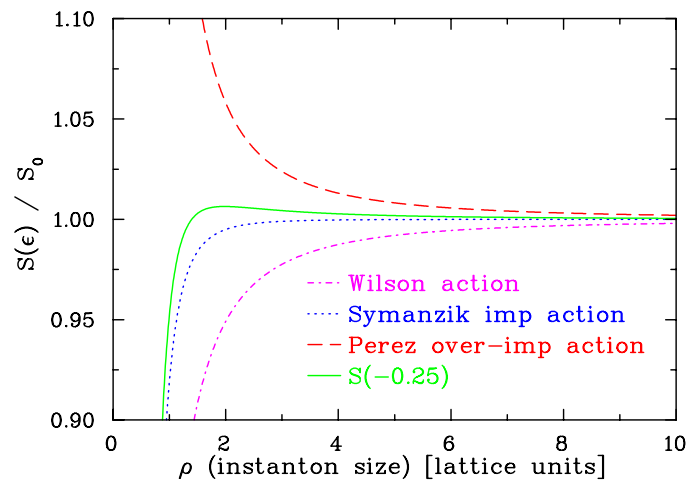

Figure 1: $S(\varepsilon) / S_{0}$ versus the instanton size, $\rho$, for the Wilson action, Symanzik-improved action, Perez over-improved action, and our over-improved action $S(-0.25) . S_{0}$ is the action for a single instanton. To preserve instantons the ideal smoothing action would give a straight line at $S(\varepsilon) / S_{0}=1$. The slope of each curve dictates how an instanton in the gauge field will evolve under smearing. Our action should be the most stable because it is mostly flat and has a dislocation threshold, given by its maximum, at $\rho \sim 1.5$.

\subsection{Topological Charge Density Correlator}

Recent studies of vacuum structure in Lattice QCD $[9,10]$ have revolved around the use of the overlap topological charge density operator [11]. The overlap operator has the benefi t of producing an integer topological charge and was fi rst to reveal the negative topological charge density correlator, $\langle q(x) q(0)\rangle$ [10] for $x>0$. Unfortunately, the overlap operator is very computationally intensive. Thus we examine the issue of whether a traditional smearing method can produce a negative correlator. Using the quenched gauge fi elds, we calculate the $\langle q(x) q(0)\rangle$ correlator on smoothed gauge fields with a three-loop $\mathscr{O}\left(a^{4}\right)$-improved lattice fi eld strength tensor [4]. Fig. 2 reports the results.

\subsection{Instanton-Like Objects}

Repeated application of a smearing algorithm will eventually reveal the presence of spherical instantons in a gauge fi eld. However, after only a small number of sweeps, these objects tend to be far from spherical. We now wish to investigate the similarity of these objects to instantons.

Using over-improved smearing we analyze the action density of a smeared fi eld to determine the peaks of maximum action and fit the instanton action density to our data. From this we can extract a size, $\rho$, for the instanton. We also extract the charge at the centre of the instanton-like

Table 1: Parameters of the gauge fields used for this investigation. Label denotes how we will refer to the respective lattices throughout this proceeding. For more information see [7, 8].

\begin{tabular}{lcccc}
\hline \hline label & size & $\beta$ & $a(\mathrm{fm})$ & $a m_{u, d} / a m_{s}$ \\
\hline Quenched & $28^{3} \times 96$ & 8.40 & 0.086 & - \\
Heavy & $28^{3} \times 96$ & 7.11 & 0.086 & $0.0124 / 0.031$ \\
Light & $28^{3} \times 96$ & 7.09 & 0.086 & $0.0062 / 0.031$ \\
\hline
\end{tabular}



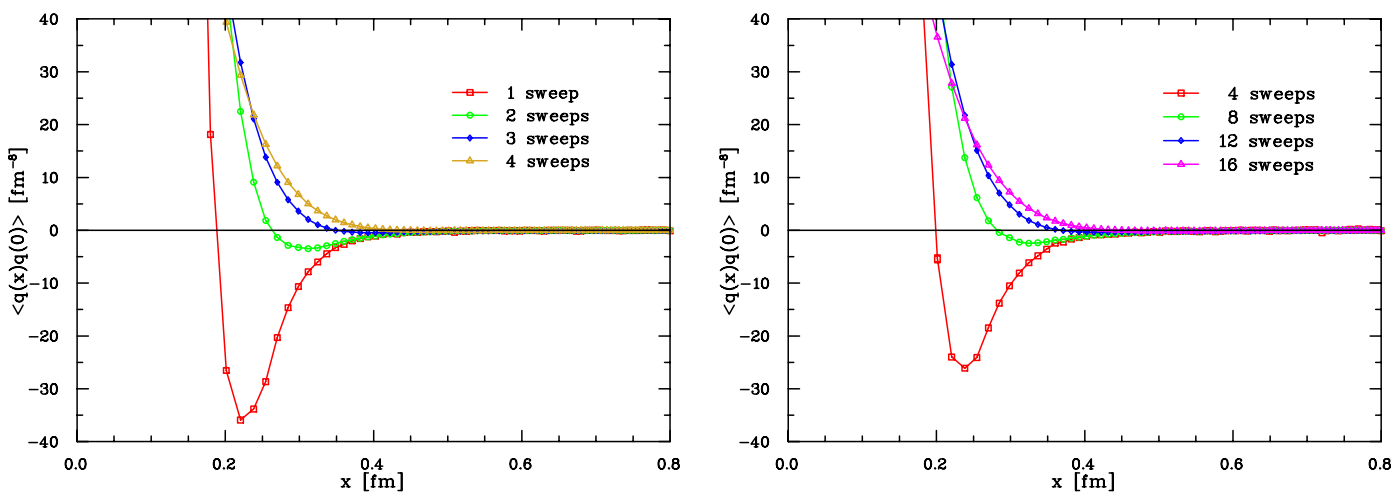

Figure 2: The topological charge density correlator $\langle q(x) q(0)\rangle$ as computed on the quenched gauge configurations for both 3-loop improved cooling (left) and over-improved stout-link smearing (right). We see that for a small number of sweeps it is possible to obtain a negative $\langle q(x) q(0)\rangle$ correlator, similar to the recent overlap results [10]. Note that errors were calculated using a first-order jackknife procedure but are too small to see.
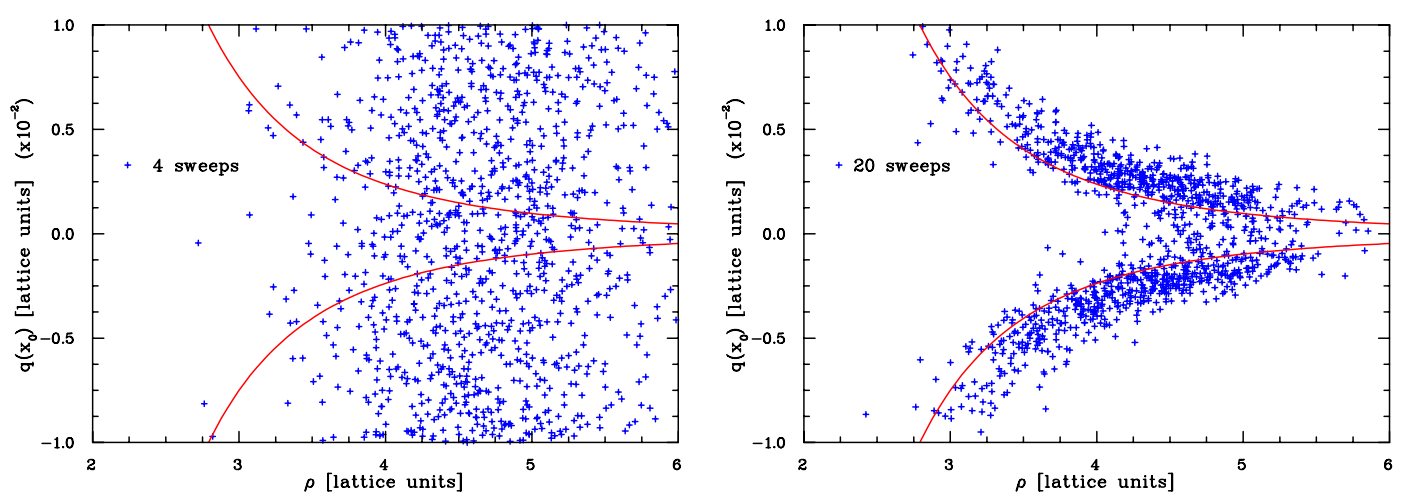

Figure 3: $q\left(x_{0}\right)$ versus the instanton size $\rho$ for 4 and 20 sweeps of over-improved stout-link smearing. Calculation details are in the text. We see that for 4 sweeps of smearing the peaks do not seem to represent instantons, but that as we smooth further the points start to cluster around the predicted line.

object, $q\left(x_{0}\right)$. Thus, if there is good agreement between the extracted $q\left(x_{0}\right)$ and that predicted by $\rho$, then we can say that the object is locally representative of an instanton.

In Fig. 3 we plot $q\left(x_{0}\right)$ vs $\rho$ for a quenched gauge fi eld after both 4 and 20 sweeps of overimproved stout-link smearing. Each cross represents a peak in the action density. If the peak were to represent an instanton then its cross should lie on the theoretical curve. We see that for 4 sweeps of smearing the peaks do not appear to represent instantons. As more UV fluctuations are removed the crosses lie closer to the line.

\subsection{The Vacuum Structure of Dynamical Gauge Fields}

Several studies of the differences in vacuum structure between quenched and dynamical fi elds have focused on the topological susceptibility

$$
\chi=\left\langle\int d^{4} x q(x) q(0)\right\rangle=\frac{\left\langle Q^{2}\right\rangle}{V}
$$




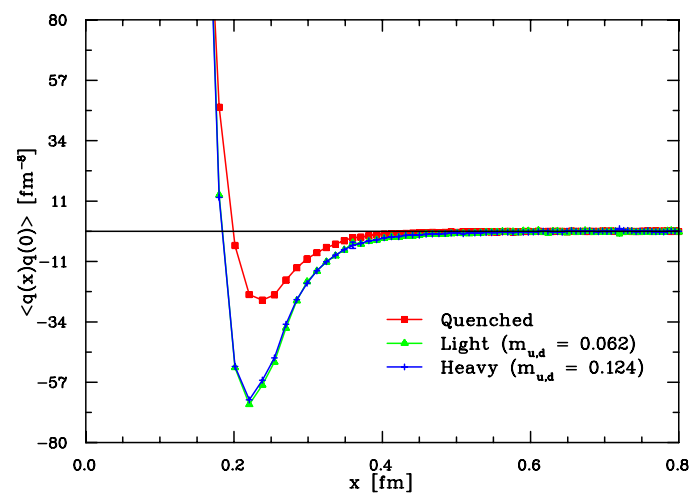

Figure 4: The topological charge density correlator $\langle q(x) q(0)\rangle$ for the quenched, light and heavy dynamicalfermion gauge fields. It is interesting to see how the dynamical fermion loops have caused the negative dip to increase in magnitude, and how this effect is greater for lighter quark masses. Although not shown, the positive contact term $\left\langle q^{2}(0)\right\rangle$ has also increased in magnitude. Exact values are given in the text.

We now extend these studies of the topological susceptibility to the $\langle q(x) q(0)\rangle$ correlator. It has been shown that [12]

$$
\left\langle\int d^{4} x q(x) q(0)\right\rangle \sim m_{\pi}^{2} \rightarrow 0 \text { in the chiral limit, }
$$

and therefore $|Q| \rightarrow 0$ also. This leads to three scenarios for how the shape of the $\langle q(x) q(0)\rangle$ could change in the presence of dynamical quarks. Either the positive contact term and the magnitude of the negative component could both increase or decrease, or they could stay the same. The only requirement is that the integral of the correlator vanishes in the chiral limit. However, as we discussed in the introduction, fermion-loop coupling renormalization leads to smaller $\beta$ admitting larger fi eld fluctuations. We therefore expect that $\left\langle q(0)^{2}\right\rangle$ should increase, and thus so must the negative component of $\langle q(x) q(0)\rangle$ increase in magnitude.

Fig. 4 shows the topological charge density correlator as calculated for the three different types of gauge fi elds. We see that the presence of dynamical quarks has caused the magnitude of the negative component of the correlator to increase, and that this effect is greater for lighter quark masses. The $x$-intercept has also moved closer towards 0 . Although not shown in the plot, the mean square density $\left\langle q^{2}(0)\right\rangle$ has also increased in magnitude. The exact values of the positive contact term are; quenched $=2924 \pm 4 \mathrm{fm}^{-8}$, heavy $=5251 \pm 12 \mathrm{fm}^{-8}$, light $=5432 \pm 8 \mathrm{fm}^{-8}$

We expect that this behaviour will be readily apparent in visualizations of the charge density, $q(x)$. Plots of $q(x)$ are shown in Fig. 5, and indeed we observe that the light and heavy gauge fi elds contain many more non-trivial fi eld fluctuations.

\section{Conclusion}

We have presented the fi rst calculation of the topological charge density correlator, $\langle q(x) q(0)\rangle$, in full QCD. Using both 3-loop improved cooling and the new over-improved stout-link smearing procedure we are able to obtain negative $\langle q(x) q(0)\rangle$ correlators. Using our proven methodology a quantitative comparison of quenched and dynamical gauge fi elds is performed. The dynamical 

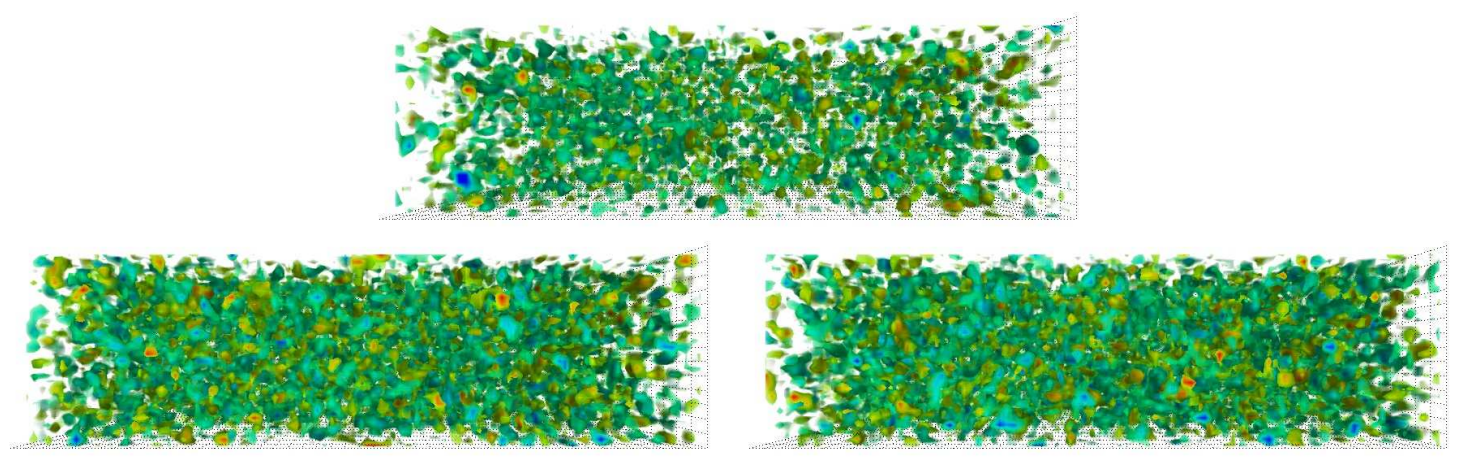

Figure 5: Topological charge densities for the quenched (top), heavy (bottom left) and light (bottom right) dynamical-fermion gauge fields. Each field has been smoothed using 4 sweeps of over-improved stoutlink smearing. We see that the bottom two dynamical gauge fields contain a higher density of non-trivial topological charge density than the quenched field. However it is difficult to see the difference between the two dynamical gauge fields.

gauge fi elds show an increase in non-trivial vacuum fi eld fluctuations. This is observed directly through visualizations of the topological charge density and via the calculation of the $\langle q(x) q(0)\rangle$ correlator. For the correlator we see an increase in the magnitude of the negative dip and positive contact term with larger effects for lighter quark masses. These observations are in accord with expectation, outlined in greater detail in a forthcoming publication [13].

\section{References}

[1] Margarita Garcia Perez et al., Nucl. Phys. B413, 535-552 (1994). [hep-lat/9309009]

[2] Colin Morningstar and Mike J. Peardon, Phys. Rev. D69, 054501 (2004). [hep-lat/0311018]

[3] Frederic D. R. Bonnet et al., Phys. Rev. D65, 114510 (2002). [hep-lat/ 0106023 ]

[4] Sundance O. Bilson-Thompson et al., Ann. Phys. 304, 1-21 (2003). [hep-lat/0203008]

[5] Sundance O. Bilson-Thompson et al., Nucl. Phys. Proc. Suppl. 109A, 116-120 (2002). [hep-lat/0112034]

[6] A. A. Belavin et al., Phys. Lett. B59, 85-87 (1975).

[7] Claude W. Bernard et al., Phys. Rev. D64, 054506 (2001). [hep-lat/ 0104002 ]

[8] C. Aubin et al., Phys. Rev. D70, 094505 (2004). [hep-lat / 0402030 ]

[9] Philippe de Forcrand, AIP Conf. Proc. 892, 29-35 (2007). [hep-lat/ 0611034 ]

[10] I. Horvath et al., Phys. Lett. B617, 49-59 (2005). [hep-lat/ 0504005 ]

[11] Herbert Neuberger, Phys. Rev. Lett. 81, 4060-4062 (1998). [hep-lat/9806025]

[12] A. Hart and M. Teper, Phys. Lett. B523, 280-292 (2001). [hep-lat/ 0108006 ]

[13] Peter J. Moran and Dererk B. Leinweber, in preparation, 2007. 